\title{
Sensomics-Assisted Flavor Decoding of Dairy Model Systems and Flavor Reconstitution Experiments
}

\author{
Florian Utz, ${ }^{1}$ Johanna Kreissl, ${ }^{2}$ Timo D. Stark, ${ }^{1}$ Christian Schmid, ${ }^{1}$ \\ Caren Tanger, ${ }^{3}$ Ulrich Kulozik, ${ }^{3}$ Thomas Hofmann, ${ }^{1}$ and Corinna Dawid ${ }^{1 *}$ \\ ${ }^{1}$ Chair for Food Chemistry and Molecular Sensory Science, Technical University of \\ Munich, Lise-Meitner-Straße 34, 85354 Freising, Germany, \\ ${ }^{2}$ Leibniz-Institute for Food Systems Biology at the Technical University of Munich, \\ Lise-Meitner-Straße 34, 85354 Freising, Germany, and \\ ${ }^{3}$ Chair of Food and Bioprocess Engineering, Technical University of Munich, \\ Weihenstephaner Berg 1, 85354 Freising, Germany
}

Running Title: Flavor blueprint of a dairy model.

* Author to whom correspondence should be addressed

PHONE +49-8161/71-2902

FAX $\quad+49-8161 / 71-2949$

E-MAIL_ corinna.dawid@tum.de 
Syntheses of long-chain $\delta$-lactones. Preparation of 5-oxo hexadecanoic acid methyl ester. Following two procedures described in literature with some modifications, ${ }^{1,2}$ 1-undecylbromid $(4.5 \mathrm{~mL} ; 20 \mathrm{mmol})$ was added dropwise to a magnesium suspension (500 $\mathrm{mg} ; 20 \mathrm{mmol})$ in dry diethyl ether $(20 \mathrm{~mL})$. The reaction was stirred for $30 \mathrm{~min}$ at room temperature and then heated under reflux for $30 \mathrm{~min}$. After cooling to room temperature, the resulting suspension was mixed with a solution of glutaric anhydride $(1.76 \mathrm{~g} ; 15 \mathrm{mmol})$ and copper $(\mathrm{l})$ iodide $(0.01 \mathrm{mmol})$ in dry diethyl ether $(20 \mathrm{~mL})$ at $-20^{\circ} \mathrm{C}$. The mixture was then stirred for $60 \mathrm{~min}$, conditioned to room temperature, and stirred again for $60 \mathrm{~min}$. To stop the reaction, hydrochloric acid $(1 \mathrm{~N} ; 40 \mathrm{~mL})$ was added. The resulting suspension was repeatedly extracted with chloroform $(3 \times 50 \mathrm{~mL}$, each), the organic phase was dried over anhydrous sodium sulfate, and, after filtration, removed from solvent in a vacuum. The raw product 5-oxo hexadecanoic acid was heated in acidified methanol $(20 \mathrm{~mL})$ at $70^{\circ} \mathrm{C}$ over night and the resulting 5-oxo hexadecanoic acid methyl ester was purified by means of medium pressure liquid chromatography (MPLC; Büchi Sepacore, Flawil, Switzerland) using a $40 \times 150 \mathrm{~mm}$ silica gel cartridge (Kinesis, Wertheim, Germany) and the following gradient of ethyl acetate (solvent A) and hexane (solvent B) with a flow of $40 \mathrm{~mL} / \mathrm{min}$ : $0 \mathrm{~min}, 100 \% \mathrm{~B} ; 5 \mathrm{~min}, 100 \% \mathrm{~B} ; 50 \mathrm{~min}$, $70 \% \mathrm{~B} ; 60 \mathrm{~min}, 30 \% \mathrm{~B} ; 65 \mathrm{~min}, 0 \% \mathrm{~B}, 70 \mathrm{~min} 0 \% \mathrm{~B}$. After solvent removal 5-oxo hexadecanoic acid methyl ester $(0.15 \mathrm{mmol} ; 0.5 \%$ in yield $)$ was obtained.

5-oxo hexadecanoic acid methyl ester: ${ }^{1} \mathrm{H}$ NMR $\left(400 \mathrm{MHz}, 25^{\circ} \mathrm{C}\right.$, chloroform-d), $\delta(\mathrm{ppm}): 3.69$ (s, 3H, H-C17); 2.49 (t, 2H, J=7.2 Hz, H-C4*); 2.40 (t, 2H, J=7.5 Hz, H$\mathrm{C6}^{*}$ ); 2.36 (t, 2H, J=7.2 Hz, H-C2); 1.92 (p, 2H, J=7.2 Hz, H-C3); 1.64-1.51 (m, 2H, $\mathrm{H}-\mathrm{C} 7) ; 1.37-1.23(\mathrm{~m}, 16 \mathrm{H}, \mathrm{H}-\mathrm{C} 8-15) ; 0.90(\mathrm{t}, 3 \mathrm{H}, \mathrm{J}=6.8 \mathrm{~Hz}, \mathrm{H}-\mathrm{C} 16) .{ }^{13} \mathrm{C}$ NMR (100 MHz, $25^{\circ} \mathrm{C}$, chloroform-d), $\delta(\mathrm{ppm}): 210.4$ (C5); 173.7 (C1); 51.6 (C17); 42.9 
(C6); 41.5 (C4); 33.1 (C2); 31.9 (C14); 29.6-29.3 (C8-13); 23.9 (C7); 22.7 (C15); 18.9 (C3); 14.1 (C16). * signals are interchangeable

Synthesis of $\delta$-hexadecalactone. The 5-oxo hexadecenoic acid methyl ester $(0.04 \mathrm{~g}$; $0.15 \mathrm{mmol})$ is reduced in a mixture consisting of methanol $(20 \mathrm{~mL})$, sodium borohydride ( $8.5 \mathrm{mg} ; 2.4 \mathrm{mmol}$ ) and monosodium phosphate $(2.5 \mathrm{mg} ; 0.18 \mathrm{mmol})$ for $5 \mathrm{~h}$ at room temperature. After addition of $10 \%$ sodium hydroxide $(100 \mathrm{~mL})$ the solution was stirred for another $1 \mathrm{~h}$ at room temperature and then adjusted to $\mathrm{pH} 1-2$ with concentrated hydrochloric acid. This solution was stirred for $1 \mathrm{~h}$ at $0{ }^{\circ} \mathrm{C}$ and extracted with diethyl ether. The combined organic layers were washed with saturated sodium chloride solution, dried over sodium sulfate and, after filtration, evaporated in a vacuum. The resulting raw product was purified by means of MPLC under the same conditions as described above. After solvent removal, $5 \mathrm{mg}$ of $\delta$ hexadecalactone $(0.02 \mathrm{mmol} ; 13 \%$ in yield $)$ were obtained.

ס-hexadecalactone: LC-MS $\left(\mathrm{ESI}^{+}\right): \mathrm{m} / \mathrm{z} 255.3\left(\mathrm{M}+\mathrm{H}^{+}\right) .{ }^{1} \mathrm{H}$ NMR $\left(400 \mathrm{MHz}, 25{ }^{\circ} \mathrm{C}\right.$, chloroform- $d), \delta(p p m): 4.27$ (m, 1H, H-C5); 2.56 (m, 1H, H-C2); 2.49-2.43 (m, 1H, H$\mathrm{C} 2) ; 1.95-1.65(\mathrm{~m}, 4 \mathrm{H}, \mathrm{H}-\mathrm{C} 3-4) ; 1.62-1.37(\mathrm{~m}, 20 \mathrm{H}, \mathrm{H}-\mathrm{C} 6-15) ; 0.92-0.85(\mathrm{~m}, 3 \mathrm{H}, \mathrm{H}-$ C16). ${ }^{13} \mathrm{C}$ NMR (100 MHz, $25^{\circ} \mathrm{C}$, chloroform-d), $\delta(\mathrm{ppm}): 172.1$ (C1); 80.8 (C5); 36.0 (C6); 32.1 (C14); 29.8-29.5 (C2, C8-13); 28.0 (C4); 25.1 (C7); 22.8 (C15); 18.7 (C3); $14.3(\mathrm{C} 16)$.

Preparation of 5-oxo octadecanoic acid methyl ester. The synthesis of 5-oxo octadecanoic acid methyl ester was analogous to the synthesis of 5-oxo hexadecanoic acid methyl ester using 1-bromotridecane as starting material in the same amount of substance. After solvent removal $80 \mathrm{mg}$ of 5-oxo octadecanoic acid methyl ester $(0.28 \mathrm{mmol} ; 1 \%$ in yield) were obtained in the last step.

5-oxo octadecanoic acid methyl ester: ${ }^{1} \mathrm{H}$ NMR $\left(400 \mathrm{MHz}, 25^{\circ} \mathrm{C}\right.$, chloroform-d), $\delta($ ppm): 3.69 (s, 3H, H-C19); 2.49 (t, 2H, J=7.2 Hz, H-C4*); 2.40 (t, 2H, J=7.5 Hz, H- 
C6*); 2.36 (t, 2H, J=7.2 Hz, H-C2); $1.92(p, 2 \mathrm{H}, J=7.2 \mathrm{~Hz}, \mathrm{H}-\mathrm{C} 3) ; 1.58$ (t, 2H, $J=7.3 \mathrm{~Hz}, \mathrm{H}-\mathrm{C} 7) ; 1.40-1.20(\mathrm{~m}, 2 \mathrm{OH}, \mathrm{H}-\mathrm{C} 8-17) ; 0.96-0.83(\mathrm{~m}, 3 \mathrm{H}, \mathrm{H}-\mathrm{C} 18) .{ }^{13} \mathrm{C}$ NMR (100 MHz, $25^{\circ} \mathrm{C}$, chloroform-d), $\delta(\mathrm{ppm}): 210.4$ (C5); 173.7 (C1); 51.6 (C19); 42.9 (C6); 41.5 (C4); 33.1 (C2); 31.9 (C16); 29.7-29.3 (C8-15); 23.9 (C7); 22.7 (C17); 18.9 (C3); 14.1 (C18). * signals are interchangeable

Synthesis of $\delta$-octadecalactone. 5-oxo octadecanoic acid methyl ester (75 mg; $0.25 \mathrm{mmol}$ ) was reduced in a mixture containing methanol $(20 \mathrm{~mL})$, sodium borohydride (18 mg; $5 \mathrm{mmol})$ and sodium monophosphate $(5 \mathrm{mg} ; 0.36 \mathrm{mmol})$ for $5 \mathrm{~h}$ at room temperature. After addition of $10 \%$ sodium hydroxide $(100 \mathrm{~mL})$ the mixture was stirred for $1 \mathrm{~h}$ at room temperature and then adjusted to $\mathrm{pH} 1-2$ with concentrated hydrochloric acid. The solution was again stirred for $1 \mathrm{~h}$ at $0{ }^{\circ} \mathrm{C}$ and extracted with diethyl ether. The combined organic layers were washed with saturated sodium chloride solution, dried over sodium sulfate and, after filtration, separated from solvent in vacuum. The resulting raw product was purified by means of MPLC under the same conditions as described above. After solvent removal, $25 \mathrm{mg}$ of $\delta$-octadecalactone $(0.08 \mathrm{mmol} ; 32 \%$ in yield $)$ were obtained.

ס-octadecalactone: LC-MS (ESI $\left.{ }^{+}\right): m / z 283.3\left(\mathrm{M}+\mathrm{H}^{+}\right) .{ }^{1} \mathrm{H}$ NMR $\left(400 \mathrm{MHz}, 25{ }^{\circ} \mathrm{C}\right.$, chloroform-d), $\delta(\mathrm{ppm}): 4.27$ (m, 1H, H-C5); 2.65-2.52 (m, 1H, H-C2); $2.44(\mathrm{~m}, 1 \mathrm{H}, \mathrm{H}-$ C2); 1.98-1.64 (m, 4H, H-C3-4); 1.63-1.41 (m, 2H, H-C6); 1.40-1.26 (m, 22H, H-C717); 0.92-0.84 (m, 3H, H-C18). ${ }^{13} \mathrm{C}$ NMR (100 MHz, $25{ }^{\circ} \mathrm{C}$, chloroform-d), $\delta(\mathrm{ppm})$ : 172.1 (C1); 80.8 (C5); 36.0 (C6); 32.1 (C16); 29.8-29.5 (C2, C8-15); 28.0 (C4); 25.1 (C7); 22.9 (C17); 18.7 (C3); 14.3 (C18).

Spectroscopic data (NMR, LC-MS) of the synthetic compounds were identical to the data obtained of the purchased $\delta$-hexa- and $\delta$-octadecalactone (aromaLAB). 


\section{REFERENCES}

(1) Naoshima, Y.; Ozawa, H.; Kondo, H.; Hayashi, S. Synthesis of racemates and $(+)$-enantiomers of $\gamma$-Caprolactone, $\gamma$-dodecanolactone and $\delta$-hexadecanolactone, lactonic sex pheromones of the dermestid beetle, rove beetle and oriental hornet, Agric. Biol. Chem. 1983, 47, pp. 1431-1434.

(2) Lhommet, G.; Fréville, S.; Thuy, V.; Petit, H.; Célérier, J. P. A general and versatile synthesis of 4- and 5-Oxoacids, Synth. Commun. 1996, 26, pp. 3897-3901. 


\section{FIGURE LEGEND}

Figure S1. Chemical structures of internal standards used for flavor quantitation of dairy products: hexanal- $d_{12}\left(1-d_{12}\right)$, acetaldehyde- $d_{3}\left(12-d_{3}\right)$, diacetyl- $d_{6}$

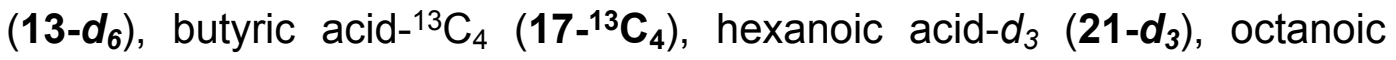

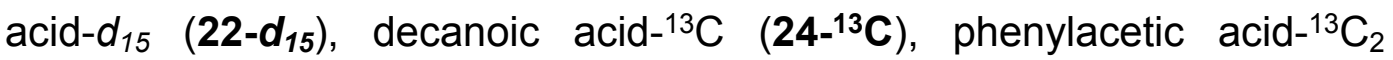
$\left(27-{ }^{13} C_{2}\right)$, vanillin- $d_{3}\left(30-d_{3}\right), \delta$-dodecalactone- $d_{2} \quad\left(\delta-C 12 L-d_{2}\right)$, dimethyl sulfide- $d_{6}\left(42-d_{6}\right)$, and acetic acid- ${ }^{13} C_{2}\left(46-{ }^{13} C_{2}\right)$. 
Utz et al., Figure S1
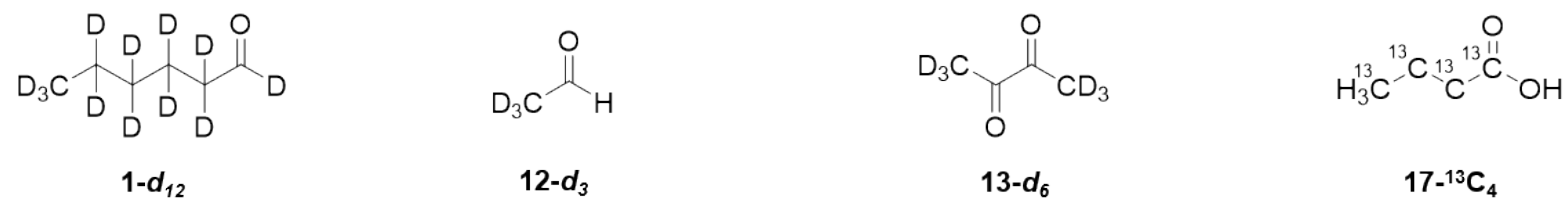<smiles>O=C(O)CCCCC#[Co]</smiles>

$21-d_{3}$

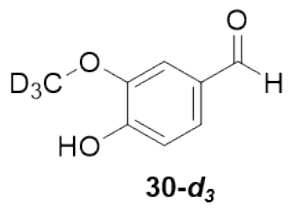

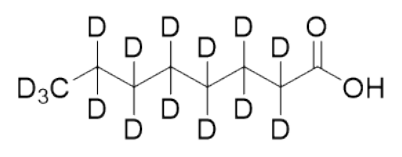

22- $d_{15}$

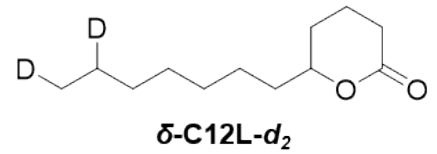

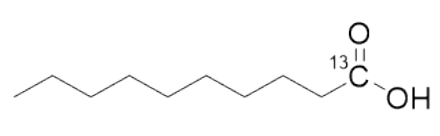

24-13 $C$

$$
\underset{42-d_{6}}{\mathrm{D}_{3} \mathrm{C}^{-\mathrm{S}} \mathrm{CD}_{3}}
$$<smiles></smiles>

$27-{ }^{13} C_{2}$

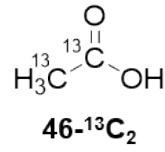


Table S1. MRM transitions of analyzed 3-NPH derivates.

\begin{tabular}{|c|c|c|c|c|c|c|c|c|}
\hline Analyte (number) qt./ql. & Ionization & $\begin{array}{c}\mathrm{Q1} \\
{[\mathrm{m} / \mathrm{z}]}\end{array}$ & $\begin{array}{c}\text { Q3 } \\
{[m / z]}\end{array}$ & $\begin{array}{c}t_{R} \\
{[\mathrm{~min}]}\end{array}$ & $\begin{array}{l}\text { DP } \\
\text { [V] }\end{array}$ & $\begin{array}{l}\text { EP } \\
{[V]}\end{array}$ & $\begin{array}{l}\text { CE } \\
{[V]}\end{array}$ & $\begin{array}{l}\text { CXP } \\
\text { [V] }\end{array}$ \\
\hline hexanal (1) qt. & $\mathrm{ESI}^{+}$ & 236.1 & 119.0 & 8.9 & 46 & 10 & 19 & 14 \\
\hline hexanal (1) ql. & $\mathrm{ESI}^{+}$ & 236.1 & 137.0 & 8.9 & 46 & 10 & 23 & 14 \\
\hline (IS) hexanal- $d_{12}\left(1-d_{12}\right) q t$. & $\mathrm{ESI}^{+}$ & 248.1 & 120.0 & 8.9 & 41 & 10 & 21 & 14 \\
\hline (IS) hexanal- $d_{12}\left(\mathbf{1}-\boldsymbol{d}_{12}\right) q l$. & $\mathrm{ESI}^{+}$ & 248.1 & 137.0 & 8.9 & 41 & 10 & 23 & 16 \\
\hline methional (2) qt. & $\mathrm{ESI}^{+}$ & 240.1 & 75.0 & 7.0 & 6 & 10 & 17 & 8 \\
\hline methional (2) ql. & $\mathrm{ESI}^{+}$ & 240.1 & 139.0 & 7.0 & 6 & 10 & 11 & 14 \\
\hline cis-3-hexenal (3) qt. & $\mathrm{ESI}^{+}$ & 234.1 & 152.0 & 8.5 & 106 & 10 & 19 & 16 \\
\hline cis-3-hexenal (3) ql. & $\mathrm{ESI}^{+}$ & 234.1 & 98.1 & 8.5 & 106 & 10 & 21 & 14 \\
\hline cis-2-nonenal (4) qt. & $\mathrm{ESI}^{+}$ & 276.2 & 139.9 & 10.4 & 156 & 10 & 25 & 18 \\
\hline cis-2-nonenal (4) ql. & $\mathrm{ESI}^{+}$ & 276.2 & 229.1 & 10.4 & 156 & 10 & 27 & 16 \\
\hline trans-2-nonenal (5) qt. & $\mathrm{ESI}^{+}$ & 276.2 & 140.1 & 10.5 & 86 & 10 & 23 & 22 \\
\hline trans-2-nonenal (5) ql. & $\mathrm{ESI}^{+}$ & 276.2 & 118.9 & 10.5 & 86 & 10 & 39 & 12 \\
\hline trans,trans-2,4-nonadienal (6) qt. & $\mathrm{ESI}^{+}$ & 274.1 & 152.0 & 10.1 & 1 & 10 & 21 & 16 \\
\hline trans,trans-2,4-nonadienal (6) ql. & $\mathrm{ESI}^{+}$ & 274.1 & 207.2 & 10.1 & 1 & 10 & 15 & 12 \\
\hline trans,cis-2,6-nonadienal (7) qt. & $\mathrm{ESI}^{+}$ & 274.1 & 119.2 & 9.8 & 1 & 10 & 27 & 12 \\
\hline trans, cis-2,6-nonadienal (7) ql. & $\mathrm{ESI}^{+}$ & 274.1 & 138.2 & 9.8 & 1 & 10 & 19 & 10 \\
\hline trans,trans-2,4-decadienal (8) qt. & $\mathrm{ESI}^{+}$ & 288.1 & 152.1 & 10.5 & 1 & 10 & 25 & 20 \\
\hline trans,trans-2,4-decadienal (8) ql. & $\mathrm{ESI}^{+}$ & 288.1 & 221.1 & 10.5 & 1 & 10 & 17 & 14 \\
\hline trans-4,5-epoxy-trans-2-decenal (9) qt. & $\mathrm{ESI}^{+}$ & 304.1 & 286.2 & 10.3 & 36 & 10 & 15 & 16 \\
\hline trans-4,5-epoxy-trans-2-decenal (9) ql. & $\mathrm{ESI}^{+}$ & 304.1 & 158.2 & 10.3 & 36 & 10 & 31 & 14 \\
\hline trans-2-undecenal (10) qt. & $\mathrm{ESI}^{+}$ & 304.2 & 118.9 & 11.3 & 1 & 10 & 31 & 20 \\
\hline trans-2-undecenal (10) ql. & $\mathrm{ESI}^{+}$ & 304.2 & 168.0 & 11.3 & 1 & 10 & 27 & 20 \\
\hline trans-2-dodecenal (11) qt. & $\mathrm{ESI}^{+}$ & 318.2 & 182.2 & 11.7 & 1 & 10 & 27 & 8 \\
\hline trans-2-dodecenal (11) ql. & $\mathrm{ESI}^{+}$ & 318.2 & 271.1 & 11.7 & 1 & 10 & 33 & 16 \\
\hline acetaldehyde (12) qt. & $\mathrm{ESI}^{+}$ & 180.0 & 137.0 & 5.0 & 1 & 10 & 17 & 16 \\
\hline acetaldehyde (12) ql. & $\mathrm{ESI}^{+}$ & 180.0 & 119.1 & 5.0 & 1 & 10 & 15 & 12 \\
\hline (IS) acetaldehyde- $d_{3}\left(12-d_{3}\right) q t$. & $\mathrm{ESI}^{+}$ & 183.0 & 136.8 & 5.0 & 6 & 10 & 17 & 18 \\
\hline (IS) acetaldehyde- $d_{3}\left(12-d_{3}\right) q l$. & $\mathrm{ESI}^{+}$ & 183.0 & 118.9 & 5.0 & 6 & 10 & 15 & 12 \\
\hline diacetyl (13) qt. & $\mathrm{ESI}^{-}$ & 219.9 & 136.9 & 5.8 & -105 & -10 & -24 & -17 \\
\hline diacetyl (13) ql. & $\mathrm{ESI}^{-}$ & 219.9 & 122.0 & 5.8 & -105 & -10 & -24 & -15 \\
\hline (IS) diacetyl- $d_{6}\left(13-d_{6}\right) q t$. & $\mathrm{ESI}^{-}$ & 226.0 & 137.8 & 5.7 & -10 & -10 & -26 & -21 \\
\hline
\end{tabular}




\begin{tabular}{|c|c|c|c|c|c|c|c|c|}
\hline (IS) diacetyl- $d_{6}\left(13-d_{6}\right) q I$. & $\mathrm{ESI}^{-}$ & 226.0 & 91.7 & 5.7 & -10 & -10 & -30 & -11 \\
\hline acetoin (14) qt. & $\mathrm{ESI}^{-}$ & 222.0 & 136.9 & 3.6 & -70 & -10 & -20 & -13 \\
\hline acetoin (14) ql. & $\mathrm{ESI}^{-}$ & 222.0 & 203.9 & 3.6 & -70 & -10 & -18 & -19 \\
\hline 1-hexen-3-one (15) qt. & $\mathrm{ESI}^{+}$ & 234.1 & 165.0 & 8.8 & 156 & 10 & 21 & 18 \\
\hline 1-hexen-3-one (15) ql. & $\mathrm{ESI}^{+}$ & 234.1 & 118.9 & 8.8 & 156 & 10 & 31 & 14 \\
\hline 1-octen-3-one (16) qt. & $\mathrm{ESI}^{+}$ & 262.1 & 165.2 & 9.9 & 56 & 10 & 27 & 20 \\
\hline 1-octen-3-one (16) ql. & $\mathrm{ESI}^{+}$ & 262.1 & 119.0 & 9.9 & 56 & 10 & 29 & 14 \\
\hline butyric acid (17) qt. & $\mathrm{ESI}^{-}$ & 222.0 & 136.8 & 2.2 & -85 & -10 & -26 & -15 \\
\hline butyric acid (17) ql. & $\mathrm{ESI}^{-}$ & 222.0 & 151.9 & 2.2 & -85 & -10 & -22 & -23 \\
\hline (IS) butyric acid- ${ }^{13} \mathrm{C}_{4}\left(1 \mathbf{1 7}^{-13} \mathrm{C}_{\mathbf{4}}\right) q t$. & $\mathrm{ESI}^{-}$ & 226.0 & 136.7 & 2.2 & -100 & -10 & -28 & -17 \\
\hline (IS) butyric acid- ${ }^{13} \mathrm{C}_{4}\left(17-{ }^{13} \mathrm{C}_{4}\right)$ ql. & $\mathrm{ESI}^{-}$ & 226.0 & 151.9 & 2.2 & -100 & -10 & -22 & -19 \\
\hline (IS) butyric acid- ${ }^{13} \mathrm{C}_{4}\left(17-{ }^{13} \mathrm{C}_{4}\right) q t$. & $\mathrm{ESI}^{+}$ & 228.1 & 137.9 & 2.2 & 86 & 10 & 23 & 20 \\
\hline (IS) butyric acid- ${ }^{13} \mathrm{C}_{4}\left(17-{ }^{13} \mathrm{C}_{4}\right) q l$. & $\mathrm{ESI}^{+}$ & 228.1 & 91.9 & 2.2 & 86 & 10 & 23 & 10 \\
\hline 2-methylbutanoic acid (18) qt. & $\mathrm{ESI}^{+}$ & 238.1 & 137.0 & 3.1 & 101 & 10 & 23 & 16 \\
\hline 2-methylbutanoic acid (18) ql. & $\mathrm{ESI}^{+}$ & 238.1 & 101.8 & 3.1 & 101 & 10 & 23 & 10 \\
\hline 3-methylbutanoic acid (19) qt. & $\mathrm{ESI}^{+}$ & 238.1 & 138.2 & 3.2 & 1 & 10 & 23 & 12 \\
\hline 3-methylbutanoic acid (19) ql. & $\mathrm{ESI}^{+}$ & 238.1 & 102.0 & 3.2 & 1 & 10 & 23 & 12 \\
\hline pentanoic acid (20) qt. & $\mathrm{ESI}^{+}$ & 238.1 & 136.9 & 3.4 & 1 & 10 & 23 & 16 \\
\hline pentanoic acid (20) ql. & $\mathrm{ESI}^{+}$ & 238.1 & 102.0 & 3.4 & 1 & 10 & 23 & 16 \\
\hline hexanoic acid (21) qt. & $\mathrm{ESI}^{-}$ & 250.0 & 137.0 & 4.9 & -125 & -10 & -36 & -15 \\
\hline hexanoic acid (21) ql. & $\mathrm{ESI}^{-}$ & 250.0 & 151.8 & 4.9 & -125 & -10 & -24 & -21 \\
\hline (IS) hexanoic acid- $d_{3}\left(21-d_{3}\right) q t$. & $\mathrm{ESI}^{-}$ & 253.1 & 136.7 & 4.8 & -100 & -10 & -26 & -35 \\
\hline (IS) hexanoic acid- $d_{3}\left(21-d_{3}\right) q l$. & $\mathrm{ESI}^{-}$ & 253.1 & 151.8 & 4.8 & -100 & -10 & -24 & -37 \\
\hline octanoic acid (22) qt. & $\mathrm{ESI}^{-}$ & 278.0 & 136.9 & 7.2 & -150 & -10 & -30 & -17 \\
\hline octanoic acid (22) ql. & $\mathrm{ESI}^{-}$ & 278.0 & 151.9 & 7.2 & -150 & -10 & -26 & -17 \\
\hline (IS) octanoic acid- $d_{15}\left(\mathbf{2 2 -} \boldsymbol{d}_{15}\right) q t$ & $\mathrm{ESI}^{-}$ & 293.1 & 136.7 & 7.1 & -120 & -10 & -34 & -11 \\
\hline (IS) octanoic acid- $d_{15}\left(\mathbf{2 2}-\boldsymbol{d}_{15}\right) \mathrm{ql}$. & $\mathrm{ESI}^{-}$ & 293.1 & 152.9 & 7.1 & -120 & -10 & -30 & -7 \\
\hline nonanoic acid (23) qt. & $\mathrm{ESI}^{-}$ & 292.0 & 136.9 & 7.9 & -110 & -10 & -30 & -19 \\
\hline nonanoic acid (23) ql. & $\mathrm{ESI}^{-}$ & 292.0 & 151.9 & 7.9 & -110 & -10 & -26 & -21 \\
\hline decanoic acid (24) qt. & $\mathrm{ESI}^{-}$ & 306.0 & 137.0 & 8.5 & -130 & -10 & -34 & -15 \\
\hline decanoic acid (24) ql. & $\mathrm{ESI}^{-}$ & 306.0 & 152.0 & 8.5 & -130 & -10 & -28 & -17 \\
\hline (IS) decanoic acid- ${ }^{13} \mathrm{C}\left(24-{ }^{13} \mathrm{C}\right) q t$. & $\mathrm{ESI}^{-}$ & 307.1 & 136.9 & 8.5 & -120 & -10 & -32 & -9 \\
\hline (IS) decanoic acid- ${ }^{13} \mathrm{C}\left(24-{ }^{13} \mathrm{C}\right) \mathrm{ql}$. & $\mathrm{ESI}^{-}$ & 307.1 & 151.7 & 8.5 & -120 & -10 & -28 & -21 \\
\hline dodecanoic acid (25) qt. & $\mathrm{ESI}^{-}$ & 334.0 & 136.9 & 9.5 & -155 & -10 & -36 & -15 \\
\hline dodecanoic acid (25) ql. & $\mathrm{ESI}^{-}$ & 334.0 & 151.9 & 9.5 & -155 & -10 & -30 & -19 \\
\hline
\end{tabular}


tetradecanoic acid (26) qt.

tetradecanoic acid (26) ql.

phenylacetic acid (27) qt.

phenylacetic acid (27) ql.

(IS) phenylacetic acid- ${ }^{13} C_{2}\left(27-{ }^{13} C_{2}\right) q t$.

(IS) phenylacetic acid- ${ }^{13} C_{2}\left(27-{ }^{13} C_{2}\right) q l$.

benzaldehyde (28) qt.

benzaldehyde (28) ql.

phenylpropanoic acid (29) qt.

phenylpropanoic acid (29) ql.

vanillin (30) $q t$.

$$
\text { vanillin (30) } q l .
$$

(IS) vanillin- $d_{3}\left(30-d_{3}\right) q t$.

(IS) vanillin- $d_{3}\left(30-d_{3}\right) q l$.

aminoacetophenone (31) qt.

aminoacetophenone (31) ql. sotolon (32) qt. sotolon (32) ql.

2-acetyl-1-pyrroline (33) qt

2-acetyl-1-pyrroline (33) ql.

2-acetyl-2-thiazoline (34) qt.

2-acetyl-2-thiazoline (34) ql. acetic acid (46) qt. acetic acid (46) ql.

(IS) acetic acid- ${ }^{13} \mathrm{C}_{2}\left(46-{ }^{13} \mathrm{C}_{2}\right) q t$.

(IS) acetic acid- ${ }^{13} C_{2}\left(46-{ }^{13} C_{2}\right) q l$.

\begin{tabular}{|c|c|c|c|}
\hline $\mathrm{ESI}^{-}$ & 362.1 & 136.9 & 10.5 \\
\hline $\mathrm{ESI}^{-}$ & 362.1 & 152.0 & 10.5 \\
\hline $\mathrm{ESI}^{+}$ & 272.1 & 91.0 & 3.8 \\
\hline $\mathrm{ESI}^{+}$ & 272.1 & 136.0 & 3.8 \\
\hline $\mathrm{ESI}^{+}$ & 274.1 & 91.9 & 3.8 \\
\hline $\mathrm{ESI}^{+}$ & 274.1 & 138.1 & 3.8 \\
\hline $\mathrm{ESI}^{+}$ & 241.9 & 106.0 & 8.1 \\
\hline $\mathrm{ESI}^{+}$ & 241.9 & 137.0 & 8.1 \\
\hline $\mathrm{ESI}^{+}$ & 286.1 & 105.0 & 5.0 \\
\hline $\mathrm{ESI}^{+}$ & 286.1 & 90.8 & 5.0 \\
\hline $\mathrm{ESI}^{+}$ & 288.0 & 151.0 & 6.4 \\
\hline $\mathrm{ESI}^{+}$ & 288.0 & 105.0 & 6.4 \\
\hline $\mathrm{ESI}^{+}$ & 291.0 & 154.2 & 6.4 \\
\hline $\mathrm{ESI}^{+}$ & 291.0 & 108.9 & 6.4 \\
\hline $\mathrm{ESI}^{+}$ & 270.9 & 133.9 & 6.6 \\
\hline $\mathrm{ESI}^{+}$ & 270.9 & 92.9 & 6.6 \\
\hline $\mathrm{ESI}^{-}$ & 262.0 & 136.9 & 4.4 \\
\hline $\mathrm{ESI}^{-}$ & 262.0 & 106.8 & 4.4 \\
\hline $\mathrm{ESI}^{-}$ & 398.2 & 137.0 & 6.1 \\
\hline $\mathrm{ESI}^{-}$ & 398.2 & 203.0 & 6.1 \\
\hline $\mathrm{ESI}^{+}$ & 265.0 & 138.0 & 3.9 \\
\hline $\mathrm{ESI}^{+}$ & 265.0 & 131.9 & 3.9 \\
\hline $\mathrm{ESI}^{-}$ & 193.9 & 152.0 & 1.1 \\
\hline $\mathrm{ESI}^{-}$ & 193.9 & 137.0 & 1.1 \\
\hline $\mathrm{ESI}^{-}$ & 195.9 & 151.9 & 1.1 \\
\hline $\mathrm{ESI}^{-}$ & 195.9 & 136.9 & 1.1 \\
\hline
\end{tabular}

\begin{tabular}{c}
-160 \\
-160 \\
1 \\
1 \\
76 \\
76 \\
61 \\
61 \\
1 \\
1 \\
1 \\
1 \\
41 \\
41 \\
1 \\
1 \\
-5 \\
-5 \\
-200 \\
-200 \\
1 \\
1 \\
-100 \\
-100 \\
-30 \\
-30 \\
\hline $110 n$
\end{tabular}

$\begin{array}{r}-10 \\ -10 \\ 10 \\ 10 \\ 10 \\ 10 \\ 10 \\ 10 \\ 10 \\ 10 \\ 10 \\ 10 \\ 10 \\ 10 \\ 10 \\ 10 \\ -10 \\ -10 \\ -10 \\ -10 \\ 10 \\ 10 \\ -10 \\ -10 \\ -10 \\ -10 \\ \hline-\end{array}$

$-17$

$-17$

10

16

10

16

12

14

48

16

18

12

18

12

14

10

$-5$

$-19$

$-8$

$-8$

$\mathbf{E P}=$ entrance potential. $\mathbf{C E}=$ collision energy. $\mathbf{C X P}=$ cell exit potential. 
Table S2. MRM transitions of $\delta$-lactones and the corresponding 5-hydroxy acids.

\begin{tabular}{|c|c|c|c|c|c|c|c|c|}
\hline Analyte (number) qt./ql. & Ionization & $\begin{array}{c}\text { Q1 } \\
{[\mathrm{m} / \mathrm{z}]}\end{array}$ & $\begin{array}{c}\text { Q3 } \\
{[\mathrm{m} / \mathrm{z}]}\end{array}$ & $\begin{array}{c}t_{R} \\
\text { [min] }\end{array}$ & $\begin{array}{l}\text { DP } \\
\text { [V] }\end{array}$ & $\begin{array}{l}\text { EP } \\
\text { [V] }\end{array}$ & $\begin{array}{l}\text { CE } \\
\text { [V] }\end{array}$ & $\begin{array}{c}\text { CXP } \\
\text { [V] }\end{array}$ \\
\hline (IS) $\delta$-dodecalactone- $d_{2}\left(\delta\right.$-C12L-d $\left.{ }_{2}\right) q t$ & $\mathrm{ESI}^{+}$ & 201.0 & 165.0 & 1.7 & 91 & 10 & 13 & 10 \\
\hline (IS) $\delta$-dodecalactone-d $d_{2}\left(\delta-C 12 L-d_{2}\right) q l$. & $\mathrm{ESI}^{+}$ & 201.0 & 55.0 & 1.7 & 91 & 10 & 37 & 8 \\
\hline 5-hydroxy dodecanoic acid- $d_{2} q t$. & $\mathrm{ESI}^{+}$ & 219.1 & 184.1 & 1.7 & 61 & 10 & 15 & 6 \\
\hline 5-hydroxy dodecanoic acid- $d_{2} q l$. & $\mathrm{ESI}^{+}$ & 219.1 & 166.0 & 1.7 & 61 & 10 & 19 & 8 \\
\hline$\delta$-tetradecalactone (35) qt. & $\mathrm{ESI}^{+}$ & 227.2 & 209.1 & 1.9 & 51 & 10 & 9 & 26 \\
\hline$\delta$-tetradecalactone (35) ql. & $\mathrm{ESI}^{+}$ & 227.2 & 69.0 & 1.9 & 51 & 10 & 23 & 8 \\
\hline 5-hydroxy tetradecanoic acid (36) qt. & $\mathrm{ESI}^{+}$ & 245.2 & 210.2 & 1.9 & 1 & 10 & 15 & 12 \\
\hline 5-hydroxy tetradecanoic acid (36) ql. & $\mathrm{ESI}^{+}$ & 245.2 & 192.2 & 1.9 & 1 & 10 & 17 & 10 \\
\hline$\delta$-hexadecalactone (37) qt. & $\mathrm{ESI}^{+}$ & 255.2 & 55.0 & 2.0 & 46 & 10 & 45 & 8 \\
\hline$\delta$-hexadecalactone (37) ql. & $\mathrm{ESI}^{+}$ & 255.2 & 41.0 & 2.0 & 46 & 10 & 65 & 18 \\
\hline 5-hydroxy hexadecanoic acid (38) qt. & $\mathrm{ESI}^{+}$ & 273.3 & 256.2 & 2.0 & 1 & 10 & 9 & 16 \\
\hline 5-hydroxy hexadecanoic acid (38) ql. & $\mathrm{ESI}^{+}$ & 273.3 & 238.2 & 2.0 & 1 & 10 & 15 & 14 \\
\hline$\delta$-octadecalactone (39) qt. & $\mathrm{ESI}^{+}$ & 283.2 & 69.0 & 2.2 & 51 & 10 & 33 & 8 \\
\hline$\delta$-octadecalactone (39) ql. & $\mathrm{ESI}^{+}$ & 283.2 & 97.1 & 2.2 & 51 & 10 & 23 & 12 \\
\hline 5-hydroxy octadecanoic acid (40) qt. & $\mathrm{ESI}^{+}$ & 301.3 & 266.2 & 2.2 & 1 & 10 & 15 & 16 \\
\hline 5-hydroxy octadecanoic acid (40) ql. & $\mathrm{ESI}^{+}$ & 301.3 & 248.3 & 2.2 & 1 & 10 & 19 & 16 \\
\hline
\end{tabular}

qt. = quantifier MRM. ql. = qualifier MRM. Q1 = precursor ion. Q3 = selected product ion. $\mathbf{t}_{\mathbf{R}}=$ retention time. $\mathbf{D P}=$ declustering potential.

$\mathbf{E P}=$ entrance potential. $\mathbf{C E}=$ collision energy. $\mathbf{C X P}=$ cell exit potential. 